Supporting Information

Structural Optimization of Triboelectric Nanogenerator for Harvesting Water Wave Energy

Tao Jiang, ${ }^{a \perp}$ Li Min Zhang, ${ }^{a \perp}$ Xiangyu Chen, ${ }^{a}$ Chang Bao Han,,${ }^{a}$ Wei Tang,,${ }^{a}$ Chi Zhang, ${ }^{a}$ Liang Xu, ${ }^{a}$ and Zhong Lin Wang ${ }^{* a b}$

${ }^{a}$ Beijing Institute of Nanoenergy and Nanosystems, Chinese Academy of Sciences, Beijing 100083, China

${ }^{b}$ School of Materials Science and Engineering, Georgia Institute of Technology, Atlanta, GA 30332-0245,

USA

*E-mail: zlwang@gatech.edu.

${ }^{\perp}$ These authors contribute equally to this work. 


\section{Determination of $x_{1}-t$ relationship during the collision between TENG and ball}

Based on Newton's second law and Hooke's law, the $x_{1}(t)$ equations were derived for two cases of $x_{1 \mathrm{~m}} \leq x_{1 \mathrm{c}}$ and $x_{1 \mathrm{~m}}>x_{1 \mathrm{c}}$ in three collision modes. Note that after the collision between metal ball and acrylic, they have the same velocity $v_{1}$, and the very short collision time is neglected. We marked the time at initial undeformed position $\left(x_{1}=0\right)$ as the zero time $(t=0)$. After $t=0$, the moving ball and acrylic can be considered as one object suffering a drag force from an ideal spring (wavy-structure core).

\section{(1) Horizontal collision}

In the horizontal collision, for $x_{1 \mathrm{~m}} \leq x_{1 \mathrm{c}}$, we can establish the following equations

$$
\begin{aligned}
& F=-k_{1} x_{1}(\mathrm{~S} 1), a=\frac{F}{m+m_{0}}=-\frac{k_{1} x_{1}}{m+m_{0}}(\mathrm{~S} 2), \quad x_{1}=\int v(t) d t(\mathrm{~S} 3), \quad v(t=0)=v_{1}(\mathrm{~S} 4), x_{1}(t=0)=0(\mathrm{~S} 5), \\
& a(t=0)=0 \quad(\mathrm{~S} 6), \quad v(t)=v_{1}+\int a d t=v_{1}-\frac{k_{1}}{m+m_{0}} \int x_{1} d t=v_{1}-\frac{k_{1}}{m+m_{0}} \int\left(\int v(t) d t\right) d t
\end{aligned}
$$

where $F, a, v$ respectively represent the resultant force, acceleration, and velocity of the object consisting of ball and acrylic. By the differentiation of both sides of Equation (S7) twice, we obtain

$v^{\prime \prime}(t)+\frac{k_{1}}{m+m_{0}} v(t)=0$

The general solution of Equation (S8) is given by

$$
v(t)=C_{1} \cos \sqrt{\frac{k_{1}}{m+m_{0}}} t+C_{2} \sin \sqrt{\frac{k_{1}}{m+m_{0}}} t
$$

Then the $a$ - $t$ relationship is obtained from the differentiation of $v(t)$ by $t$

$$
a(t)=-C_{1} \sqrt{\frac{k_{1}}{m+m_{0}}} \sin \sqrt{\frac{k_{1}}{m+m_{0}}} t+C_{2} \sqrt{\frac{k_{1}}{m+m_{0}}} \cos \sqrt{\frac{k_{1}}{m+m_{0}}} t
$$

Combining Equations (S9), (S4), (S10), and (S6), we can get $C_{1}=v_{1}, C_{2}=0$. Then, the $v(t)$ and $x_{1}(t)$ are given by

$$
v(t)=v_{1} \cos \sqrt{\frac{k_{1}}{m+m_{0}}} t
$$


$x_{1}(t)=v_{1} \sqrt{\frac{m+m_{0}}{k_{1}}} \sin \sqrt{\frac{k_{1}}{m+m_{0}}} t$

For the case of $x_{1 \mathrm{~m}}>x_{1 \mathrm{c}}$, when $0 \leq t \leq t_{\mathrm{c}}$, the spring constant does not change, and the derivation of $x_{1}(t)$ is the same as the case of $x_{1 \mathrm{~m}} \leq x_{1 \mathrm{c}}$. Also the $x_{1}(t)$ equation is the same as Equation (S12). After $t_{\mathrm{c}}$, the spring constant is transformed from $k_{1}$ to $k_{2}$. When $t_{\mathrm{c}} \leq t \leq 2 t_{\mathrm{m}}-t_{\mathrm{c}}$, the object consisting of ball and acrylic obeys the following equations

$$
\begin{aligned}
& F=-k_{1} x_{1 \mathrm{c}}-k_{2} \Delta x_{1}(\mathrm{~S} 13), \quad a=\frac{F}{m+m_{0}}=-\frac{k_{1} x_{1 \mathrm{c}}}{m+m_{0}}-\frac{k_{2} \Delta x_{1}}{m+m_{0}} \quad(\mathrm{~S} 14), \quad \Delta x_{1}=\int_{t_{\mathrm{c}}}^{t} v(t) d t \\
& v\left(t=t_{\mathrm{c}}\right)=v_{\mathrm{c}} \quad(\mathrm{S} 16), \quad \Delta x_{1}\left(t=t_{\mathrm{c}}\right)=0 \quad(\mathrm{~S} 17), \quad a\left(t=t_{\mathrm{c}}\right)=-\frac{k_{1} x_{1 \mathrm{c}}}{m+m_{0}} \\
& v(t)=v_{\mathrm{c}}+\int_{t_{\mathrm{c}}}^{t} a d t=v_{\mathrm{c}}-\frac{k_{1} x_{1 \mathrm{c}}\left(t-t_{\mathrm{c}}\right)}{m+m_{0}}-\frac{k_{2}}{m+m_{0}} \int_{t_{\mathrm{c}}}^{t}\left(\int_{t_{\mathrm{c}}}^{t} v(t) d t\right) d t
\end{aligned}
$$

where $\Delta x_{1}$ represents the further displacement after $x_{1 \mathrm{c}}$. By the differentiation of both sides of Equation (S19) twice, we obtain

$$
v^{\prime \prime}(t)+\frac{k_{2}}{m+m_{0}} v(t)=0
$$

The $v(t)$ and $a(t)$ are given by

$$
\begin{aligned}
& v(t)=C_{1} \cos \sqrt{\frac{k_{2}}{m+m_{0}}} t+C_{2} \sin \sqrt{\frac{k_{2}}{m+m_{0}}} t \\
& a(t)=-C_{1} \sqrt{\frac{k_{2}}{m+m_{0}}} \sin \sqrt{\frac{k_{2}}{m+m_{0}}} t+C_{2} \sqrt{\frac{k_{2}}{m+m_{0}}} \cos \sqrt{\frac{k_{2}}{m+m_{0}}} t
\end{aligned}
$$

By combining Equations (S21), (S16), (S22), and (S18), we can get

$$
\begin{aligned}
& C_{1}=v_{\mathrm{c}} \cos \sqrt{\frac{k_{2}}{m+m_{0}}} t_{\mathrm{c}}+\frac{k_{1} x_{1 \mathrm{c}}}{\sqrt{\left(m+m_{0}\right) k_{2}}} \sin \sqrt{\frac{k_{2}}{m+m_{0}}} t_{\mathrm{c}} \\
& C_{2}=v_{\mathrm{c}} \sin \sqrt{\frac{k_{2}}{m+m_{0}}} t_{\mathrm{c}}-\frac{k_{1} x_{1 \mathrm{c}}}{\sqrt{\left(m+m_{0}\right) k_{2}}} \cos \sqrt{\frac{k_{2}}{m+m_{0}}} t_{\mathrm{c}}
\end{aligned}
$$

Therefore, the $v(t)$ and $x_{1}(t)$ are given by

$$
v(t)=v_{\mathrm{c}} \cos \sqrt{\frac{k_{2}}{m+m_{0}}}\left(t-t_{\mathrm{c}}\right)-\frac{k_{1} x_{1 \mathrm{c}}}{\sqrt{\left(m+m_{0}\right) k_{2}}} \sin \sqrt{\frac{k_{2}}{m+m_{0}}}\left(t-t_{\mathrm{c}}\right)
$$


$x_{1}(t)=v_{\mathrm{c}} \sqrt{\frac{m+m_{0}}{k_{2}}} \sin \sqrt{\frac{k_{2}}{m+m_{0}}}\left(t-t_{\mathrm{c}}\right)+\frac{k_{1} x_{1 \mathrm{c}}}{k_{2}} \cos \sqrt{\frac{k_{2}}{m+m_{0}}}\left(t-t_{\mathrm{c}}\right)-\frac{k_{1} x_{1 \mathrm{c}}}{k_{2}}+x_{1 \mathrm{c}}$

In these equations, the velocity $v_{\mathrm{c}}$ at the $x_{1 \mathrm{c}}$, and the time $t_{\mathrm{c}}$ and $t_{\mathrm{m}}$ when compressed to the $x_{1 \mathrm{c}}$ and $x_{1 \mathrm{~m}}$ are determined as follows. First, $t_{\mathrm{c}}$ is calculated by $x_{1}\left(t=t_{\mathrm{c}}\right)=x_{1 \mathrm{c}}$ and Equation (S12), which is given by

$t_{\mathrm{c}}=\sqrt{\frac{m+m_{0}}{k_{1}}} \arcsin \frac{x_{1 \mathrm{c}}}{v_{1}} \sqrt{\frac{k_{1}}{m+m_{0}}}$

Second, $v_{\mathrm{c}}$ is calculated by $v\left(t=t_{\mathrm{c}}\right)=v_{\mathrm{c}}$ and $x_{1}\left(t=t_{\mathrm{c}}\right)=x_{1 \mathrm{c}}$, given by

$v_{\mathrm{c}}=\sqrt{v_{1}^{2}-\frac{k_{1}}{m+m_{0}} x_{1 \mathrm{c}}^{2}}$

Third, $t_{\mathrm{m}}$ is obtained by $v\left(t=t_{\mathrm{m}}\right)=0$ and Equation (S25)

$t_{\mathrm{m}}=\sqrt{\frac{m+m_{0}}{k_{2}}} \arctan \frac{v_{\mathrm{c}} \sqrt{\left(m+m_{0}\right) k_{2}}}{k_{1} x_{1 \mathrm{c}}}+t_{\mathrm{c}}$

When $2 t_{\mathrm{m}}-t_{\mathrm{c}} \leq t \leq 2 t_{\mathrm{m}}$, the following equations are obtained

$$
\begin{aligned}
& F=-k_{1} x_{1 \mathrm{c}}-k_{1} \Delta x_{1}(\mathrm{~S} 30), \quad a=\frac{F}{m+m_{0}}=-\frac{k_{1} x_{1 \mathrm{c}}}{m+m_{0}}-\frac{k_{1} \Delta x_{1}}{m+m_{0}} \quad(\mathrm{~S} 31), \quad \Delta x_{1}=\int_{2 t_{\mathrm{m}}-t_{\mathrm{c}}}^{t} v(t) d t \\
& v\left(t=2 t_{\mathrm{m}}-t_{\mathrm{c}}\right)=-v_{\mathrm{c}}(\mathrm{S} 33), \quad \Delta x_{1}\left(t=2 t_{\mathrm{m}}-t_{\mathrm{c}}\right)=0 \quad(\mathrm{~S} 34), \quad a\left(t=2 t_{\mathrm{m}}-t_{\mathrm{c}}\right)=-\frac{k_{1} x_{1 \mathrm{c}}}{m+m_{0}} \\
& v(t)=-v_{\mathrm{c}}+\int_{2 t_{\mathrm{m}}-t_{\mathrm{c}}}^{t} a d t=-v_{\mathrm{c}}-\frac{k_{1} x_{1 \mathrm{c}}\left(t-2 t_{\mathrm{m}}+t_{\mathrm{c}}\right)}{m+m_{0}}-\frac{k_{1}}{m+m_{0}} \int_{2 t_{\mathrm{m}}-t_{\mathrm{c}}}^{t}\left(\int_{2 t_{\mathrm{m}}-t_{\mathrm{c}}}^{t} v(t) d t\right) d t
\end{aligned}
$$

Then similar to the above method, we obtain

$$
\begin{aligned}
& v(t)=C_{1} \cos \sqrt{\frac{k_{1}}{m+m_{0}}} t+C_{2} \sin \sqrt{\frac{k_{1}}{m+m_{0}}} t \\
& a(t)=-C_{1} \sqrt{\frac{k_{1}}{m+m_{0}}} \sin \sqrt{\frac{k_{1}}{m+m_{0}}} t+C_{2} \sqrt{\frac{k_{1}}{m+m_{0}}} \cos \sqrt{\frac{k_{1}}{m+m_{0}}} t
\end{aligned}
$$

where $C_{1}$ and $C_{2}$ are given by

$$
C_{1}=-v_{\mathrm{c}} \cos \sqrt{\frac{k_{1}}{m+m_{0}}}\left(2 t_{\mathrm{m}}-t_{\mathrm{c}}\right)+x_{1 \mathrm{c}} \sqrt{\frac{k_{1}}{m+m_{0}}} \sin \sqrt{\frac{k_{1}}{m+m_{0}}}\left(2 t_{\mathrm{m}}-t_{\mathrm{c}}\right)
$$


$C_{2}=-v_{\mathrm{c}} \sin \sqrt{\frac{k_{1}}{m+m_{0}}}\left(2 t_{\mathrm{m}}-t_{\mathrm{c}}\right)-x_{1 \mathrm{c}} \sqrt{\frac{k_{1}}{m+m_{0}}} \cos \sqrt{\frac{k_{1}}{m+m_{0}}}\left(2 t_{\mathrm{m}}-t_{\mathrm{c}}\right)$

Then, the $v(t)$ and $x_{1}(t)$ are given by

$$
\begin{aligned}
& v(t)=-v_{\mathrm{c}} \cos \sqrt{\frac{k_{1}}{m+m_{0}}}\left(t-2 t_{\mathrm{m}}+t_{\mathrm{c}}\right)-x_{1 \mathrm{c}} \sqrt{\frac{k_{1}}{m+m_{0}}} \sin \sqrt{\frac{k_{1}}{m+m_{0}}}\left(t-2 t_{\mathrm{m}}+t_{\mathrm{c}}\right) \\
& x_{1}(t)=-v_{\mathrm{c}} \sqrt{\frac{m+m_{0}}{k_{1}}} \sin \sqrt{\frac{k_{1}}{m+m_{0}}}\left(t-2 t_{\mathrm{m}}+t_{\mathrm{c}}\right)+x_{\mathrm{lc}} \cos \sqrt{\frac{k_{1}}{m+m_{0}}}\left(t-2 t_{\mathrm{m}}+t_{\mathrm{c}}\right)
\end{aligned}
$$

\section{(2) Vertical collision}

In the vertical collision, the gravity force should be included. The top point of the metal ball was kept at the same height $h$, so the ball velocity $v_{0}$ when it contacts with the surface of acrylic can be obtained by $v_{0}=\sqrt{2 g(h-d)}$. After the collision between metal ball and acrylic, they have the same velocity $v_{1}$. For $x_{1 \mathrm{~m}} \leq x_{1 \mathrm{c}}$, the following equations are established

$$
\begin{aligned}
& F=\left(m+m_{0}\right) g-k_{1} x_{1} \quad(\mathrm{~S} 43), \quad a=\frac{F}{m+m_{0}}=g-\frac{k_{1} x_{1}}{m+m_{0}} \quad(\mathrm{~S} 44), \quad x_{1}=\int v(t) d t \\
& v(t=0)=v_{1} \quad(\mathrm{~S} 46), \quad x_{1}(t=0)=0 \quad(\mathrm{~S} 47), \quad a(t=0)=g \\
& v(t)=v_{1}+\int a d t=v_{1}+g t-\frac{k_{1}}{m+m_{0}} \int\left(\int v(t) d t\right) d t
\end{aligned}
$$

By the differentiation of both sides of Equation (S49) twice, and according to Equations (S46) and (S48), we obtain the equations for $v(t)$ and $x_{1}(t)$

$$
\begin{aligned}
& v(t)=v_{1} \cos \sqrt{\frac{k_{1}}{m+m_{0}}} t+g \sqrt{\frac{m+m_{0}}{k_{1}}} \sin \sqrt{\frac{k_{1}}{m+m_{0}}} t \\
& x_{1}(t)=v_{1} \sqrt{\frac{m+m_{0}}{k_{1}}} \sin \sqrt{\frac{k_{1}}{m+m_{0}}} t-\frac{\left(m+m_{0}\right) g}{k_{1}} \cos \sqrt{\frac{k_{1}}{m+m_{0}}} t+\frac{\left(m+m_{0}\right) g}{k_{1}}
\end{aligned}
$$

For the case of $x_{1 \mathrm{~m}}>x_{1 \mathrm{c}}$, when $0 \leq t \leq t_{\mathrm{c}}$, the $x_{1}(t)$ equation is the same as Equation (S51). When $t_{\mathrm{c}} \leq$ $t \leq 2 t_{\mathrm{m}}-t_{\mathrm{c}}$, we established the following equations

$$
F=\left(m+m_{0}\right) g-k_{1} x_{1 \mathrm{c}}-k_{2} \Delta x_{1}(\mathrm{~S} 52), \quad a=\frac{F}{m+m_{0}}=g-\frac{k_{1} x_{1 \mathrm{c}}}{m+m_{0}}-\frac{k_{2} \Delta x_{1}}{m+m_{0}} \quad(\mathrm{~S} 53), \quad \Delta x_{1}=\int_{t_{\mathrm{c}}}^{t} v(t) d t(\mathrm{~S} 54)
$$




$$
\begin{aligned}
& v\left(t=t_{\mathrm{c}}\right)=v_{\mathrm{c}} \quad(\mathrm{S} 55), \quad \Delta x_{1}\left(t=t_{\mathrm{c}}\right)=0 \quad(\mathrm{~S} 56), \quad a\left(t=t_{\mathrm{c}}\right)=g-\frac{k_{1} x_{1 \mathrm{c}}}{m+m_{0}} \\
& v(t)=v_{\mathrm{c}}+\int_{t_{\mathrm{c}}}^{t} a d t=v_{\mathrm{c}}+\left(g-\frac{k_{1} x_{1 \mathrm{c}}}{m+m_{0}}\right)\left(t-t_{\mathrm{c}}\right)-\frac{k_{2}}{m+m_{0}} \int_{t_{\mathrm{c}}}^{t}\left(\int_{t_{\mathrm{c}}}^{t} v(t) d t\right) d t
\end{aligned}
$$

Utilizing similar method, we can obtain the $v(t)$ and $x_{1}(t)$ equations

$$
\begin{aligned}
v(t)= & v_{\mathrm{c}} \cos \sqrt{\frac{k_{2}}{m+m_{0}}}\left(t-t_{\mathrm{c}}\right)+\frac{\left(m+m_{0}\right) g-k_{1} x_{1 \mathrm{c}}}{\sqrt{\left(m+m_{0}\right) k_{2}}} \sin \sqrt{\frac{k_{2}}{m+m_{0}}}\left(t-t_{\mathrm{c}}\right) \\
x_{1}(t)= & v_{\mathrm{c}} \sqrt{\frac{m+m_{0}}{k_{2}}} \sin \sqrt{\frac{k_{2}}{m+m_{0}}}\left(t-t_{\mathrm{c}}\right)-\frac{\left(m+m_{0}\right) g-k_{1} x_{1 \mathrm{c}}}{k_{2}} \cos \sqrt{\frac{k_{2}}{m+m_{0}}}\left(t-t_{\mathrm{c}}\right) \\
& +\frac{\left(m+m_{0}\right) g-k_{1} x_{1 \mathrm{c}}}{k_{2}}+x_{1 \mathrm{c}}
\end{aligned}
$$

The velocity $v_{\mathrm{c}}$ is calculated by $v\left(t=t_{\mathrm{c}}\right)=v_{\mathrm{c}}, x_{1}\left(t=t_{\mathrm{c}}\right)=x_{1 \mathrm{c}}$, and Equations (S50) and (S51), given by

$$
v_{\mathrm{c}}=\sqrt{v_{1}^{2}+2 g x_{1 \mathrm{c}}-\frac{k_{1}}{m+m_{0}} x_{1 \mathrm{c}}^{2}}
$$

The $t_{\mathrm{c}}$ is calculated by $x_{1}\left(t=t_{\mathrm{c}}\right)=x_{1 \mathrm{c}}$ and Equation (S51), given by

$$
t_{\mathrm{c}}=\sqrt{\frac{m+m_{0}}{k_{1}}}\left[\arcsin \frac{k_{1} x_{1 \mathrm{c}}-\left(m+m_{0}\right) g}{\sqrt{\left(m+m_{0}\right)^{2} g^{2}+\left(m+m_{0}\right) k_{1} v_{1}^{2}}}+\arcsin \frac{\left(m+m_{0}\right) g}{\sqrt{\left(m+m_{0}\right)^{2} g^{2}+\left(m+m_{0}\right) k_{1} v_{1}^{2}}}\right]
$$

The $t_{\mathrm{m}}$ is obtained by $v\left(t=t_{\mathrm{m}}\right)=0$ and Equation (S59)

$$
t_{\mathrm{m}}=\sqrt{\frac{m+m_{0}}{k_{2}}} \arctan \frac{v_{\mathrm{c}} \sqrt{\left(m+m_{0}\right) k_{2}}}{k_{1} x_{1 \mathrm{c}}-\left(m+m_{0}\right) g}+t_{\mathrm{c}}
$$

When $2 t_{\mathrm{m}}-t_{\mathrm{c}} \leq t \leq 2 t_{\mathrm{m}}$, the following equations can be obtained

$$
\begin{aligned}
& F=\left(m+m_{0}\right) g-k_{1} x_{1 \mathrm{c}}-k_{1} \Delta x_{1} \quad(\mathrm{~S} 64), a=\frac{F}{m+m_{0}}=g-\frac{k_{1} x_{1 \mathrm{c}}}{m+m_{0}}-\frac{k_{1} \Delta x_{1}}{m+m_{0}}(\mathrm{~S} 65), \Delta x_{1}=\int_{2 t_{\mathrm{m}}-t_{\mathrm{c}}}^{t} v(t) d t(\mathrm{~S} 66) \\
& v\left(t=2 t_{\mathrm{m}}-t_{\mathrm{c}}\right)=-v_{\mathrm{c}}(\mathrm{S} 67), \quad \Delta x_{1}\left(t=2 t_{\mathrm{m}}-t_{\mathrm{c}}\right)=0 \quad(\mathrm{~S} 68), \quad a\left(t=2 t_{\mathrm{m}}-t_{\mathrm{c}}\right)=g-\frac{k_{1} x_{1 \mathrm{c}}}{m+m_{0}} \quad(\mathrm{~S} 69) \\
& v(t)=-v_{\mathrm{c}}+\int_{2 t_{\mathrm{m}}-t_{\mathrm{c}}}^{t} a d t=-v_{\mathrm{c}}+\left(g-\frac{k_{1} x_{1 \mathrm{c}}}{m+m_{0}}\right)\left(t-2 t_{\mathrm{m}}+t_{\mathrm{c}}\right)-\frac{k_{1}}{m+m_{0}} \int_{2 t_{\mathrm{m}}-t_{\mathrm{c}}}^{t}\left(\int_{2 t_{\mathrm{m}}-t_{\mathrm{c}}}^{t} v(t) d t\right) d t
\end{aligned}
$$

Then the $v(t)$ and $x_{1}(t)$ equations are obtained by 


$$
\begin{aligned}
v(t)= & -v_{\mathrm{c}} \cos \sqrt{\frac{k_{1}}{m+m_{0}}}\left(t-2 t_{\mathrm{m}}+t_{\mathrm{c}}\right)+\frac{\left(m+m_{0}\right) g-k_{1} x_{1 \mathrm{c}}}{\sqrt{\left(m+m_{0}\right) k_{1}}} \sin \sqrt{\frac{k_{1}}{m+m_{0}}}\left(t-2 t_{\mathrm{m}}+t_{\mathrm{c}}\right) \\
x_{1}(t)= & -v_{\mathrm{c}} \sqrt{\frac{m+m_{0}}{k_{1}}} \sin \sqrt{\frac{k_{1}}{m+m_{0}}}\left(t-2 t_{\mathrm{m}}+t_{\mathrm{c}}\right)+\frac{k_{1} x_{1 \mathrm{c}}-\left(m+m_{0}\right) g}{k_{1}} \cos \sqrt{\frac{k_{1}}{m+m_{0}}}\left(t-2 t_{\mathrm{m}}+t_{\mathrm{c}}\right) \\
& +\frac{\left(m+m_{0}\right) g}{k_{1}}
\end{aligned}
$$

\section{(3) Sloping collision}

In the sloping collision, the gravity force can be divided into two components, i.e., the forces along the ramp and perpendicular to the ramp. The component perpendicular to the ramp is offset by the normal force of the ramp. The component force along the ramp will act on the acrylic and wavestructured core. If the sloping angle is defined as $\theta$, the component force $F_{1}$ is $\left(m+m_{0}\right) g \sin \theta$ for the object consisting of metal ball and acrylic. By comparing with the vertical collision, this is the only difference. So the equations of $v(t)$ and $x_{1}(t)$ can be obtained by simply substituting $g \sin \theta$ for $g$. The $v_{1}$ after the collision of ball and acrylic is given by $v_{1}=m \sqrt{2 g \sin \theta\left(L^{\prime}-d\right)} /\left(m+m_{0}\right)$. For $x_{1 \mathrm{~m}} \leq x_{1 \mathrm{c}}$, the $v(t)$ and $x_{1}(t)$ relationships are given by

$$
\begin{aligned}
& v(t)=v_{1} \cos \sqrt{\frac{k_{1}}{m+m_{0}}} t+g \sin \theta \sqrt{\frac{m+m_{0}}{k_{1}}} \sin \sqrt{\frac{k_{1}}{m+m_{0}}} t \\
& x_{1}(t)=v_{1} \sqrt{\frac{m+m_{0}}{k_{1}}} \sin \sqrt{\frac{k_{1}}{m+m_{0}}} t-\frac{\left(m+m_{0}\right) g \sin \theta}{k_{1}} \cos \sqrt{\frac{k_{1}}{m+m_{0}}} t+\frac{\left(m+m_{0}\right) g \sin \theta}{k_{1}}
\end{aligned}
$$

For $x_{1 \mathrm{~m}}>x_{1 \mathrm{c}}$, when $0 \leq t \leq t_{\mathrm{c}}$, the $v(t)$ and $x_{1}(t)$ equations are the same. When $t_{\mathrm{c}} \leq t \leq 2 t_{\mathrm{m}}-t_{\mathrm{c}}$, they are

$$
\begin{aligned}
v(t)= & v_{\mathrm{c}} \cos \sqrt{\frac{k_{2}}{m+m_{0}}}\left(t-t_{\mathrm{c}}\right)+\frac{\left(m+m_{0}\right) g \sin \theta-k_{1} x_{1 \mathrm{c}}}{\sqrt{\left(m+m_{0}\right) k_{2}}} \sin \sqrt{\frac{k_{2}}{m+m_{0}}}\left(t-t_{\mathrm{c}}\right) \\
x_{1}(t)= & v_{\mathrm{c}} \sqrt{\frac{m+m_{0}}{k_{2}}} \sin \sqrt{\frac{k_{2}}{m+m_{0}}}\left(t-t_{\mathrm{c}}\right)-\frac{\left(m+m_{0}\right) g \sin \theta-k_{1} x_{1 \mathrm{c}}}{k_{2}} \cos \sqrt{\frac{k_{2}}{m+m_{0}}}\left(t-t_{\mathrm{c}}\right) \\
& +\frac{\left(m+m_{0}\right) g \sin \theta-k_{1} x_{1 \mathrm{c}}}{k_{2}}+x_{1 \mathrm{c}}
\end{aligned}
$$

When $2 t_{\mathrm{m}}-t_{\mathrm{c}} \leq t \leq 2 t_{\mathrm{m}}$, they are given by

$$
v(t)=-v_{\mathrm{c}} \cos \sqrt{\frac{k_{1}}{m+m_{0}}}\left(t-2 t_{\mathrm{m}}+t_{\mathrm{c}}\right)+\frac{\left(m+m_{0}\right) g \sin \theta-k_{1} x_{1 \mathrm{c}}}{\sqrt{\left(m+m_{0}\right) k_{1}}} \sin \sqrt{\frac{k_{1}}{m+m_{0}}}\left(t-2 t_{\mathrm{m}}+t_{\mathrm{c}}\right)
$$




$$
\begin{aligned}
x_{1}(t)= & -v_{\mathrm{c}} \sqrt{\frac{m+m_{0}}{k_{1}}} \sin \sqrt{\frac{k_{1}}{m+m_{0}}}\left(t-2 t_{\mathrm{m}}+t_{\mathrm{c}}\right)+\frac{k_{1} x_{1 \mathrm{c}}-\left(m+m_{0}\right) g \sin \theta}{k_{1}} \cos \sqrt{\frac{k_{1}}{m+m_{0}}}\left(t-2 t_{\mathrm{m}}+t_{\mathrm{c}}\right) \\
& +\frac{\left(m+m_{0}\right) g \sin \theta}{k_{1}}
\end{aligned}
$$

where $v_{\mathrm{c}}, t_{\mathrm{c}}, t_{\mathrm{m}}$ are given by

$$
\begin{aligned}
& v_{\mathrm{c}}=\sqrt{v_{1}^{2}+2 g \sin \theta x_{1 \mathrm{c}}-\frac{k_{1}}{m+m_{0}} x_{1 \mathrm{c}}^{2}} \\
& t_{\mathrm{c}}=\sqrt{\frac{m+m_{0}}{k_{1}}}\left[\arcsin \frac{k_{1} x_{1 \mathrm{c}}-\left(m+m_{0}\right) g \sin \theta}{\sqrt{\left(m+m_{0}\right)^{2} g^{2}+\left(m+m_{0}\right) k_{1} v_{1}^{2}}}+\arcsin \frac{\left(m+m_{0}\right) g \sin \theta}{\sqrt{\left(m+m_{0}\right)^{2} g^{2}+\left(m+m_{0}\right) k_{1} v_{1}^{2}}}\right] \\
& t_{\mathrm{m}}=\sqrt{\frac{m+m_{0}}{k_{2}}} \arctan \frac{v_{\mathrm{c}} \sqrt{\left(m+m_{0}\right) k_{2}}}{k_{1} x_{1 \mathrm{c}}-\left(m+m_{0}\right) g \sin \theta}+t_{\mathrm{c}}
\end{aligned}
$$

\section{Dynamic output characteristics of TENG during its collision with a metal ball}

For different collision modes, the $Q-t, I-t, V$ - $t$ relationship equations are similar. When $x_{1 \mathrm{~m}} \leq x_{1 \mathrm{c}}$, these equations are derived by solving a differential equation (Equation 2 in the text) based on interpolation results, and they are provided as Equations (9)-(11). For the case of $x_{1 \mathrm{~m}}>x_{1 \mathrm{c}}$, when $0 \leq$ $t \leq t_{\mathrm{c}}$, because the boundary condition of $Q(t=0)=0$ is the same as the case of $x_{1 \mathrm{~m}} \leq x_{1 \mathrm{c}}$, the $Q-t, I-t$, and $V-t$ have the same equations. When $t_{\mathrm{c}} \leq t \leq 2 t_{\mathrm{m}}-t_{\mathrm{c}}$, the boundary condition for solving the differential equation becomes $Q\left(t=t_{\mathrm{c}}\right)=Q\left(t_{\mathrm{c}}\right)$. Then the $Q-t, I-t$, and $V$ - $t$ relationships are given by

$$
\begin{aligned}
& Q=\exp \left(-\int_{t_{\mathrm{c}}}^{t} \frac{d t}{R C\left(x_{1}(t)\right)}\right) \times\left[Q\left(t_{\mathrm{c}}\right)+\int_{t_{\mathrm{c}}}^{t} \frac{V_{\mathrm{OC}}\left(x_{1}(t)\right)}{R} \exp \left(\int_{t_{\mathrm{c}}}^{t} \frac{d z}{R C\left(x_{1}(z)\right)}\right) d t\right] \\
& I=\frac{V_{\mathrm{OC}}\left(x_{1}(t)\right)}{R}-\frac{\exp \left(-\int_{t_{\mathrm{c}}}^{t} \frac{d t}{R C\left(x_{1}(t)\right)}\right)}{R C\left(x_{1}(t)\right)} \times\left[Q\left(t_{\mathrm{c}}\right)+\int_{t_{\mathrm{c}}}^{t} \frac{V_{\mathrm{OC}}\left(x_{1}(t)\right)}{R} \exp \left(\int_{t_{\mathrm{c}}}^{t} \frac{d z}{R C\left(x_{1}(z)\right)}\right) d t\right] \\
& V=V_{\mathrm{OC}}\left(x_{1}(t)\right)-\frac{\exp \left(-\int_{t_{\mathrm{c}}}^{t} \frac{d t}{R C\left(x_{1}(t)\right)}\right)}{C\left(x_{1}(t)\right)} \times\left[Q\left(t_{\mathrm{c}}\right)+\int_{t_{\mathrm{c}}}^{t} \frac{V_{\mathrm{OC}}\left(x_{1}(t)\right)}{R} \exp \left(\int_{t_{\mathrm{c}}}^{t} \frac{d z}{R C\left(x_{1}(z)\right)}\right) d t\right]
\end{aligned}
$$

When $2 t_{\mathrm{m}}-t_{\mathrm{c}} \leq t \leq 2 t_{\mathrm{m}}$, by applying the boundary condition of $Q\left(t=2 t_{\mathrm{m}}-t_{\mathrm{c}}\right)=Q\left(2 t_{\mathrm{m}}-t_{\mathrm{c}}\right)$, the output characteristics can be obtained as follows 


$$
\begin{aligned}
& Q=\exp \left(-\int_{2 t_{\mathrm{m}}-t_{\mathrm{c}}}^{t} \frac{d t}{R C\left(x_{1}(t)\right)}\right) \times\left[Q\left(2 t_{\mathrm{m}}-t_{\mathrm{c}}\right)+\int_{2 t_{\mathrm{m}}-t_{\mathrm{c}}}^{t} \frac{V_{\mathrm{OC}}\left(x_{1}(t)\right)}{R} \exp \left(\int_{2 t_{\mathrm{m}}-t_{\mathrm{c}}}^{t} \frac{d z}{R C\left(x_{1}(z)\right)}\right) d t\right] \\
& I=\frac{V_{\mathrm{OC}}\left(x_{1}(t)\right)}{R}-\frac{\exp \left(-\int_{2 t_{\mathrm{m}}-t_{\mathrm{c}}}^{t} \frac{d t}{R C\left(x_{1}(t)\right)}\right)}{R C\left(x_{1}(t)\right)} \times\left[Q\left(2 t_{\mathrm{m}}-t_{\mathrm{c}}\right)+\int_{2 t_{\mathrm{m}}-t_{\mathrm{c}}}^{t} \frac{V_{\mathrm{OC}}\left(x_{1}(t)\right)}{R} \exp \left(\int_{2 t_{\mathrm{m}}-t_{\mathrm{c}}}^{t} \frac{d z}{R C\left(x_{1}(z)\right)}\right) d t\right] \\
& V=V_{\mathrm{OC}}\left(x_{1}(t)\right)-\frac{\exp \left(-\int_{2 t_{\mathrm{m}}-t_{\mathrm{c}}}^{t} \frac{d t}{C\left(x_{1}(t)\right)}\right)}{R C\left(x_{1}(t)\right)} \times\left[Q\left(2 t_{\mathrm{m}}-t_{\mathrm{c}}\right)+\int_{2 t_{\mathrm{m}}-t_{\mathrm{c}}}^{t} \frac{V_{\mathrm{OC}}\left(x_{1}(t)\right)}{R} \exp \left(\int_{2 t_{\mathrm{m}}-t_{\mathrm{c}}}^{t} \frac{d z}{R C\left(x_{1}(z)\right)}\right) d t\right]
\end{aligned}
$$

After the collision $\left(t>2 t_{\mathrm{m}}\right)$, the wavy structure is fully recovered to its initial state $\left(x_{1}=0\right)$. The Equation (2) in the text can be simplified as

$$
R \frac{d Q}{d t}=-\frac{1}{C(0)} \times Q
$$

where $C(0)$ is the capacitance at $x_{1}=0$ and a constant. Then with a boundary condition of $Q\left(t=2 t_{\mathrm{m}}\right)$ $=Q\left(2 t_{\mathrm{m}}\right)$, the $Q, I, V$ can be obtained by

$$
\begin{aligned}
& Q=Q\left(2 t_{\mathrm{m}}\right) \mathrm{e}^{-\frac{t-2 t_{\mathrm{m}}}{R C(0)}} \\
& I=-\frac{Q\left(2 t_{\mathrm{m}}\right)}{R C(0)} \mathrm{e}^{-\frac{t-2 t_{\mathrm{m}}}{R C(0)}}
\end{aligned}
$$

$V=-\frac{Q\left(2 t_{\mathrm{m}}\right)}{C(0)} \mathrm{e}^{-\frac{t-2 t_{\mathrm{m}}}{R(0)}}$

It indicates that the $Q, I, V$ start to decay exponentially after the collision.

Figure S1 shows the real-time output charge, current, and voltage of the wavy-structured TENG during the horizontal collision for $d=2.0 \mathrm{~cm}$ as an example. At SC condition, the $Q-t$ curve exhibits a symmetric parabolic shape, and $I_{\mathrm{SC}}$ first increases slightly to the positive peak, then decreases to its negative peak, and finally has a slight rise. After the separation between metal ball and acrylic $(t=$ $0.015 \mathrm{~s})$, the $Q, I, V$ decays exponentially. When $R$ is small, the $Q-t, I-t, V$ - $t$ curves are close to the SC curve. As $R$ increases gradually, they approach to the OC curve gradually. The relationship between current peak, voltage peak, power peak and resistance clearly indicates the three-workingregion behavior for the TENG. Note that the $V-R$ curve has a pause at a moderate $R$, which is because at smaller $R$ the $V$ peak is negative, while at larger $R$ the $V$ peak is positive. Figure S2 
presents the dynamic output charge, current, and voltage of the wavy-structured TENG as functions of the time during the vertical collision for $d=3.5 \mathrm{~cm}$ as an example. Compared to the horizontal collision, the tendencies of $Q, I$, and $V$ with respect to $R$ for the vertical collision are similar, but the difference is that the peaks of $Q_{\mathrm{SC}}$ and $I_{\mathrm{SC}}$ are sharper.
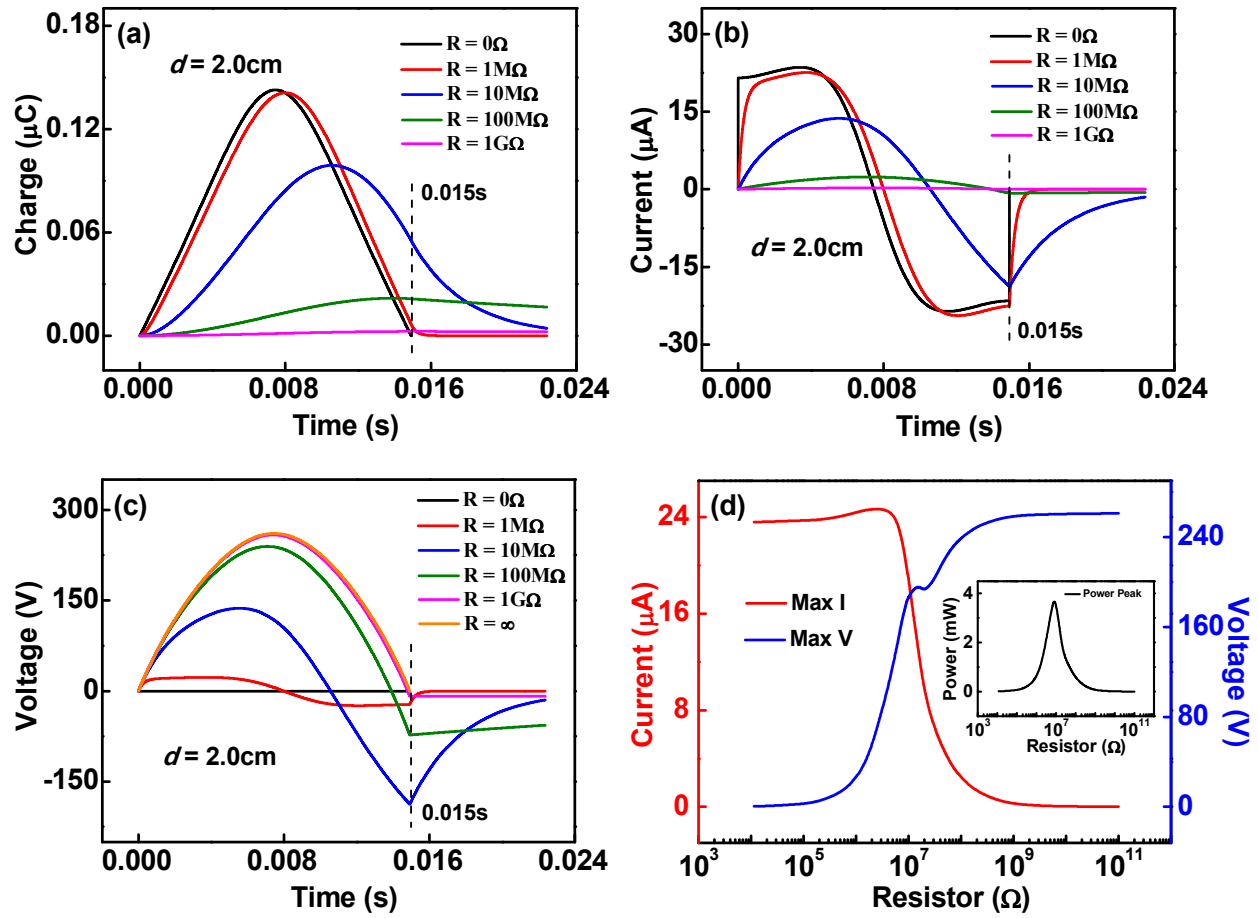

Figure S1. (a-c) Charge-time, current-time, and voltage-time relationships at different load resistances for $d=2.0 \mathrm{~cm}$ during the horizontal collision. (d) Maximum current and voltage as functions of the resistance. The insert shows the maximum output power versus resistance.
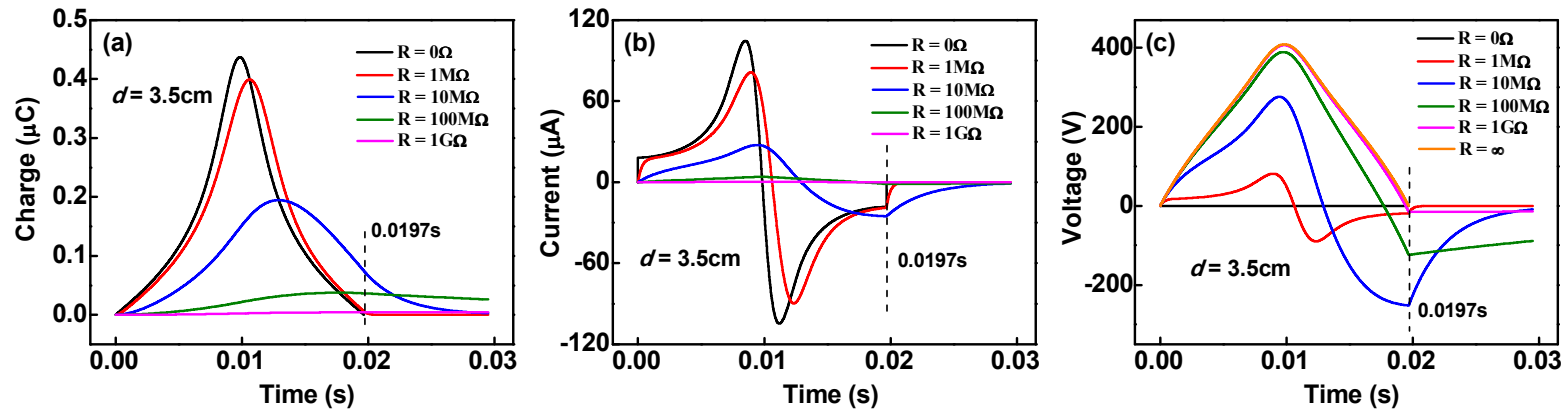

Figure S2. (a-c) Charge-time, current-time, and voltage-time relationships at different load resistances for $d=3.5 \mathrm{~cm}$ during the vertical collision. 
Figure S3 shows the maximum output power and generated electric energy of TENG as functions of ball mass for a fixed ball diameter $(d=3.0 \mathrm{~cm})$ in the vertical collision and sloping collision modes. As can be seen, the power and energy both increase with increasing the mass. That is due to the faster compression and the larger deformation depth for a larger mass at a constant allowable moving space.
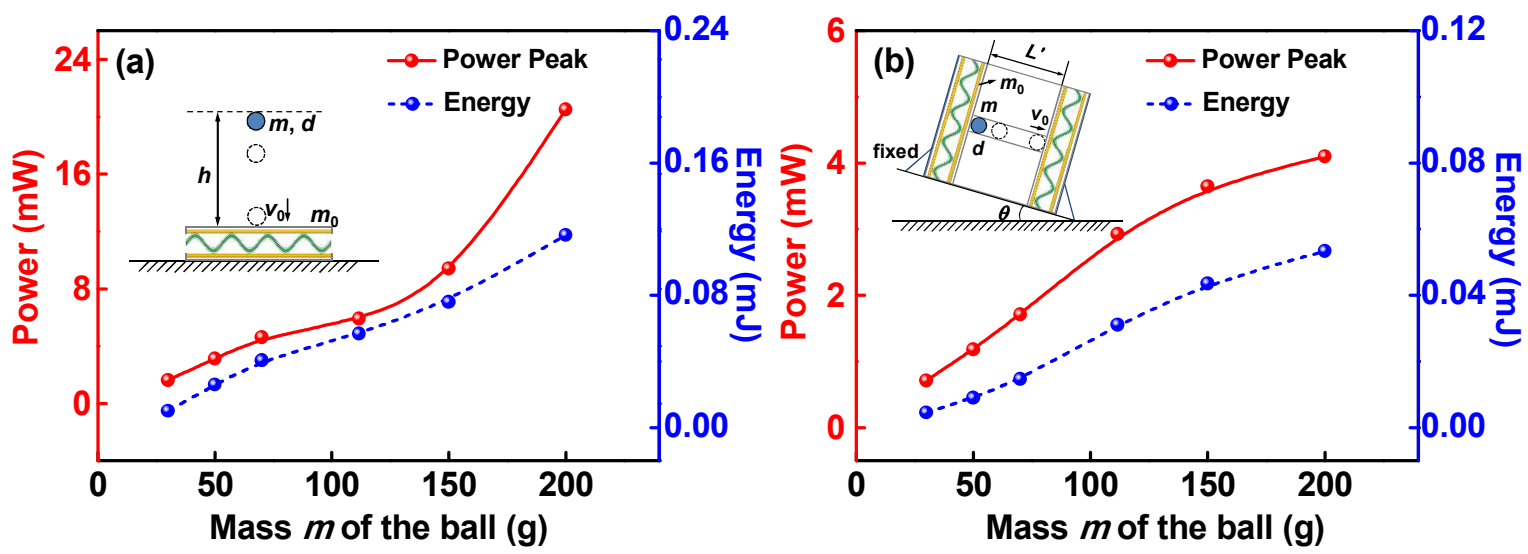

Figure S3. Effect of ball mass on the maximum power and electric energy at a fixed ball diameter $(d$ $=3.0 \mathrm{~cm}$ ): (a) in the vertical collision (b) in the sloping collision. The height $h$, moving length $L^{\prime}$, and sloping angle $\theta$ were set as $6 \mathrm{~cm}, 6 \mathrm{~cm}$, and $30^{\circ}$, respectively.

\section{Vertical collision tests and water wave tests}

(a)

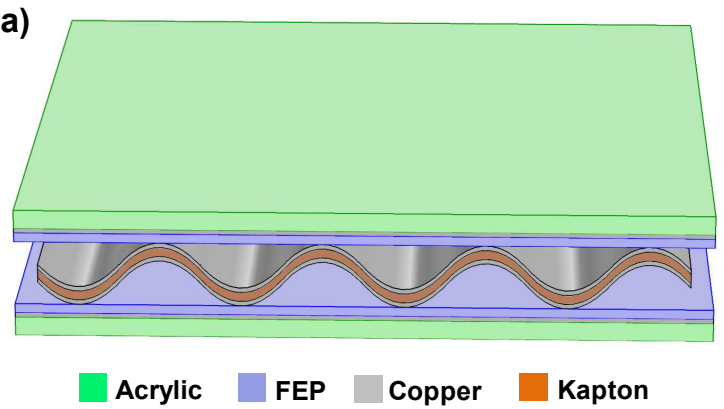

(b)

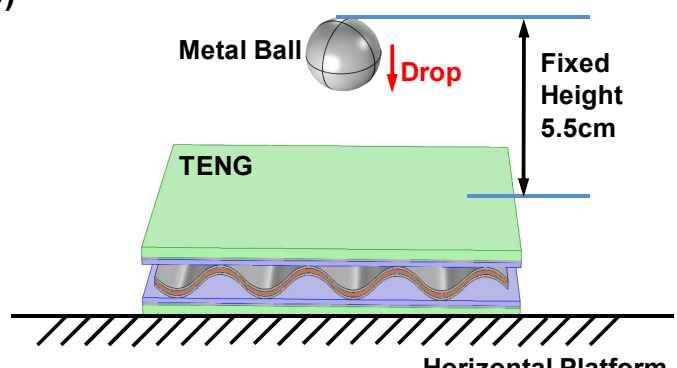

Horizontal Platform

Figure S4. (a) Structure of the wavy-structured TENG. (b) Sketch of the vertical collision experiment. 
(a)

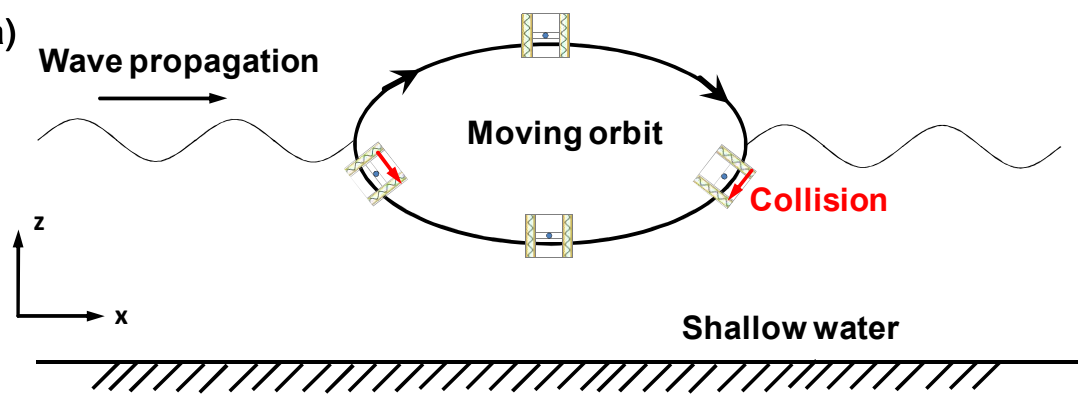

(b)
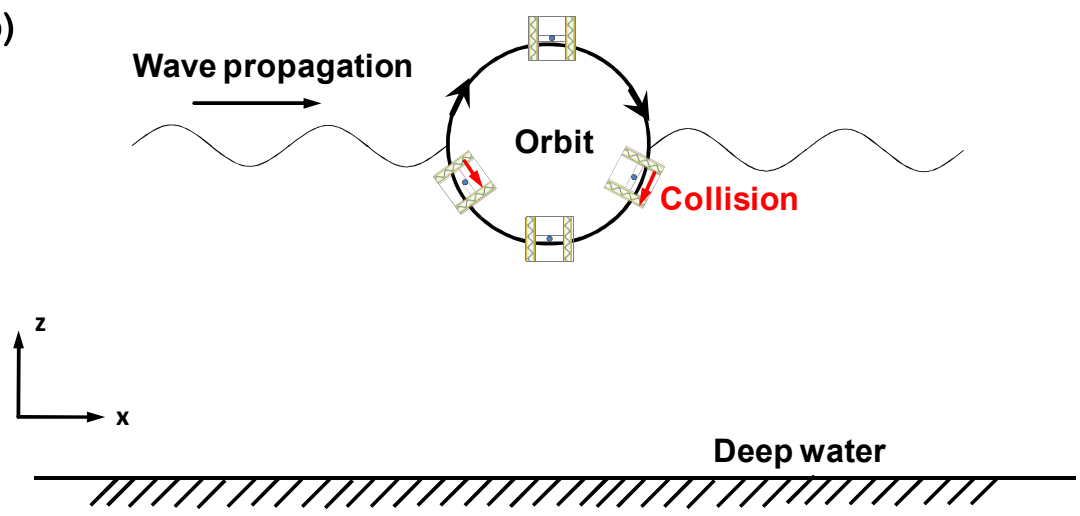

Figure S5. Schematical diagrams of the moving trajectory of TENG device containing a metal ball under the action of a single-frequency cosine wave according to Small Amplitude Wave Theory. There are two cases: (a) under shallow water; (b) under deep water.
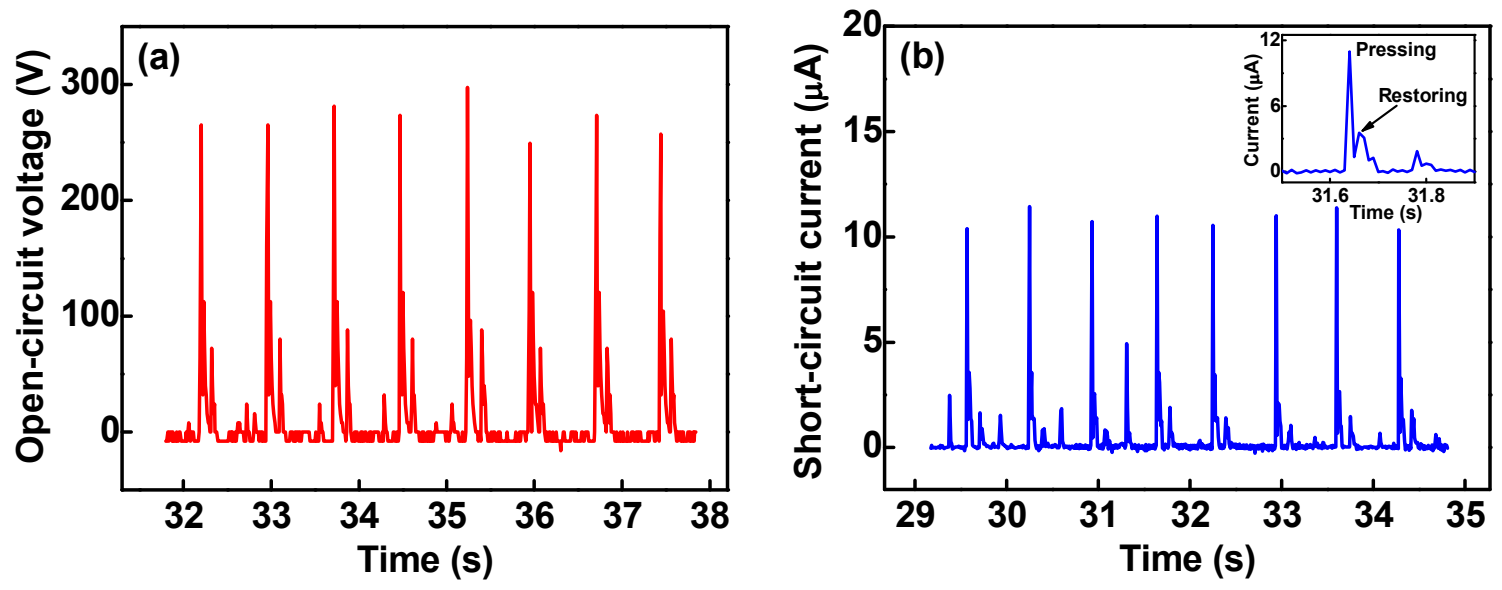

Figure S6. Enlarged view of the open-circuit voltage and short-circuit current in the water wave test for $d=3.5 \mathrm{~cm}$. 


\section{Videos}

Video S1. The device based on the wavy-structured triboelectric nanogenerators lighting up scores of LEDs agitated by the water wave motion. 